\title{
Research on the Fault Diagnosis Method of Mine Fan Based on Sound Signal Analysis
}

\author{
Shijie Song, ${ }^{1}$ Dandan Qiu ${ }^{1}{ }^{1}{ }^{1}$ and Sunwei Qin ${ }^{2}$ \\ ${ }^{1}$ School of Resources and Safety Engineering, Xingfa School of Mining Engineering, Wuhan Institute of Technology, \\ Wuhan 430073, China \\ ${ }^{2}$ Chemistry and Environmental Engineering, Wuhan Institute of Technology, Wuhan 430073, China \\ Correspondence should be addressed to Dandan Qiu; dada_qdd@163.com
}

Received 15 July 2021; Revised 8 August 2021; Accepted 13 August 2021; Published 27 August 2021

Academic Editor: Guang-Liang Feng

Copyright (C) 2021 Shijie Song et al. This is an open access article distributed under the Creative Commons Attribution License, which permits unrestricted use, distribution, and reproduction in any medium, provided the original work is properly cited.

\begin{abstract}
The underground local fan and auxiliary fan also play a vital role in the underground air quality, compared with the system fan. However, the number of underground local fans and auxiliary fans is large and widely distributed, which is disadvantageous to adopt the same method of online monitoring and fault diagnosis method as the system fan. In order to find a new fault diagnosis method, which is cost-effective and reliable, this paper proposes a fault diagnosis method based on sound signal. It analyzes the source of fan noise and studies the overall scheme of mine fan fault diagnosis expert system based on sound signal. The fault expert system consists of four parts: signal acquisition and noise elimination, feature extraction, state recognition, and fault diagnosis. Its principle is briefly introduced. The denoising method of wavelet is adopted in this paper. Wavelet packet is used to extract the characteristics of sound signal, and the energy size and energy proportion of each frequency component are used as the basis of knowledge acquisition and reasoning. Through the analysis of the measured signals of the fan in the normal operating state, the feature vectors were extracted as the basis for the discrimination of the normal state after noise elimination. At the same time, the audio processing software was used to simulate the sound signals in three fault states. Then, the feature vector of the fault state is extracted, which is obviously different from that of the fan in the normal operation. As the basis of fault state analysis of the expert system, it lays the foundation for the realization of the expert system of mine fan equipment running state diagnosis.
\end{abstract}

\section{Introduction}

Mine fan is the core equipment of mine ventilation safety, which provides the necessary power for air flow. The normal operation of the fan is very important for the whole ventilation system. At present, the real-time monitoring and fault diagnosis technology for the operation status of the main fan in the mine are relatively perfect. Compared with the local fan which is used to press in the working face and the auxiliary fan which is used to adjust the air volume in the branch air path of the mine ventilation network, the realtime monitoring of the underground auxiliary fan and the local fan has the characteristics of large number and complex distribution. Therefore, it is necessary to find an accurate and convenient detection method to detect the operation status of the fan in real time, so as to ensure a good working environment in the mine and the health and labor safety of underground workers.

Nowadays, most of the fan monitoring uses vibration signals for online monitoring. There are always one or more inherent vibration frequencies in the operation of the fan. When the fan runs abnormally, it may produce one or more frequency components different from its own natural frequency. Relevant scholars at home and abroad have done relevant research on fan monitoring and fault diagnosis based on vibration signal, temperature, and artificial intelligence. Yao and others proposed a nonlinear autoregressive moving average model to test the sunspot data and then applied the model to fan fault diagnosis, with high prediction accuracy [1]. Hong and Liao proposed a classification method based on Drosophila algorithm to optimize the least squares support vector machine (LSSVM). Based on the 
eigenvectors of fan vibration frequency domain as learning samples, the improved LSSVM model is used to identify the energy characteristics of fan vibration signal, which has the characteristics of high recognition rate and fast diagnosis speed [2]. Shu established the mine fan fault monitoring model by extracting the complex number of fault features in the fan vibration signal, combined with the principle and method of weighted support vector machine, and realized the online monitoring of mine fan fault [3]. Xuan and Li designed a set of online monitoring and fault diagnosis system for large-scale mine fan based on LabVIEW, realized real-time acquisition of fan vibration signal, monitored the operation status of equipment, and reached a diagnosis according to the vibration situation through analytic hierarchy process [4]. Zhai, through the combination of temperature change, vibration frequency, auxiliary, and troubleshooting diagnosis method, carried out fault diagnosis on several points of coal mine [5]. Zhao used BP neural network algorithm to predict the temperature of gearbox bearing, verified the feasibility and rationality of the network model, mastered the fan state, and ensured the stable and reliable operation of the unit [6]. Vibration signals need contact sensors to collect signals, and the fan itself is in the complex environment of high voltage and strong electromagnetic field, which affects the monitoring results and is not conducive to installation and maintenance.

In terms of audible sound, based on the analysis of acoustic characteristics, there is no relevant literature on mechanical fault diagnosis of mine fan equipment. Compared with the vibration signal, it is of great significance to introduce the sound signal into the condition monitoring of the fan equipment. The fan will produce different sound signals in different operation states. The sound signal under the normal operation state can be collected and analyzed to extract the detailed features, which can effectively carry out monitoring and fault diagnosis. Condition monitoring is used in fault diagnosis system, which includes two aspects: signal analysis and processing, and fault diagnosis. The purpose of signal analysis and processing is to transform the obtained information through certain methods and extract the most intuitive, sensitive, and useful feature information from different angles [7].

This paper studies the technical scheme of mine fan sound monitoring and diagnosis system. The front-end equipment is used to collect the sound signal of the normal operation of the mine fan. The wavelet denoising processing is used first and is then combined with the recognizable characteristics of the sound signal. Through the fault diagnosis expert system of the mine fan, the operation state of the fan can be effectively judged.

\section{New Program for Safety Monitoring of Mine Fan Operation}

There is a close relationship between the audio signal of the mine fan and its running state. The sound of the fan in the normal running state has its fixed time-frequency characteristics, which can be used as the basis for fault diagnosis. Therefore, the real-time acquisition of the sound signal from the fan equipment, the use of digital signal processing analysis of the current time-frequency information, can diagnose whether there is a fan failure or hidden danger. Compared with the vibration signal feature analysis, the fault diagnosis method of sound signal is a real noncontact, nonembedded, and 24-hour online monitoring method. Therefore, the real-time acquisition of acoustic signal from mine fan equipment and quantitative analysis of its variation law and mutation characteristics by digital signal processing method can diagnose faults or hidden dangers [8]. At the same time, compared with the vibration sensor, the sound sensor has advantages in price, long service life, and easy replacement. It is suitable for the dispersion of local fan and auxiliary fan in the mine, reflecting the economy based on sound monitoring. The overall block diagram of mine fan fault diagnosis system based on sound is shown in Figure 1.

The whole system is divided into four parts: signal acquisition and denoising, feature extraction, state recognition, and diagnosis decision.

Signal acquisition collects the sound generated by the normal operation of the mine local fan and selects the sound signal that can represent the working state of the equipment for feature extraction, which is used as the basis for diagnosing the equipment fault. After the completion of signal acquisition, the signal feature extraction is carried out. Acoustic signal feature extraction firstly denoises the collected sound signal and then transforms the denoised signal into energy as the signal feature by signal analysis and data processing. After feature extraction, the state recognition is carried out, and the characteristic parameters of acoustic signal obtained after signal processing are compared with the allowed parameters or discriminant parameters in the system knowledge base through the inference engine, so as to diagnose whether there is fault in its operation state and further determine the type of fault. Finally, the diagnosis system decides the measures to be taken according to the status of the diagnosis equipment and then predicts the possible development trend of the equipment according to the current signal, analyzes the trend, and makes a decision.

The monitoring and fault diagnosis of mine local fan can be realized by collecting the sound signal of fan operation, combining with the signal denoising and feature extraction technology, and then using the expert system.

\section{Acoustic Signal Acquisition and Processing}

Due to the complex environment of underground mining face, a large amount of noise will be mixed when collecting the fan signal, which is a typical nonstationary signal. Fan noise is a by-product of fan operation, but it also contains rich information about the operation process. Experienced operators can often judge the equipment status by listening to the sound changes in the operation process [9].

\subsection{Source of Fan Noise}

3.1.1. Noise of Blade Rotation. When the blade rotates, it will rub or impact with the air. The noise is sharper when the speed is faster and the frequency of air connection is higher. 


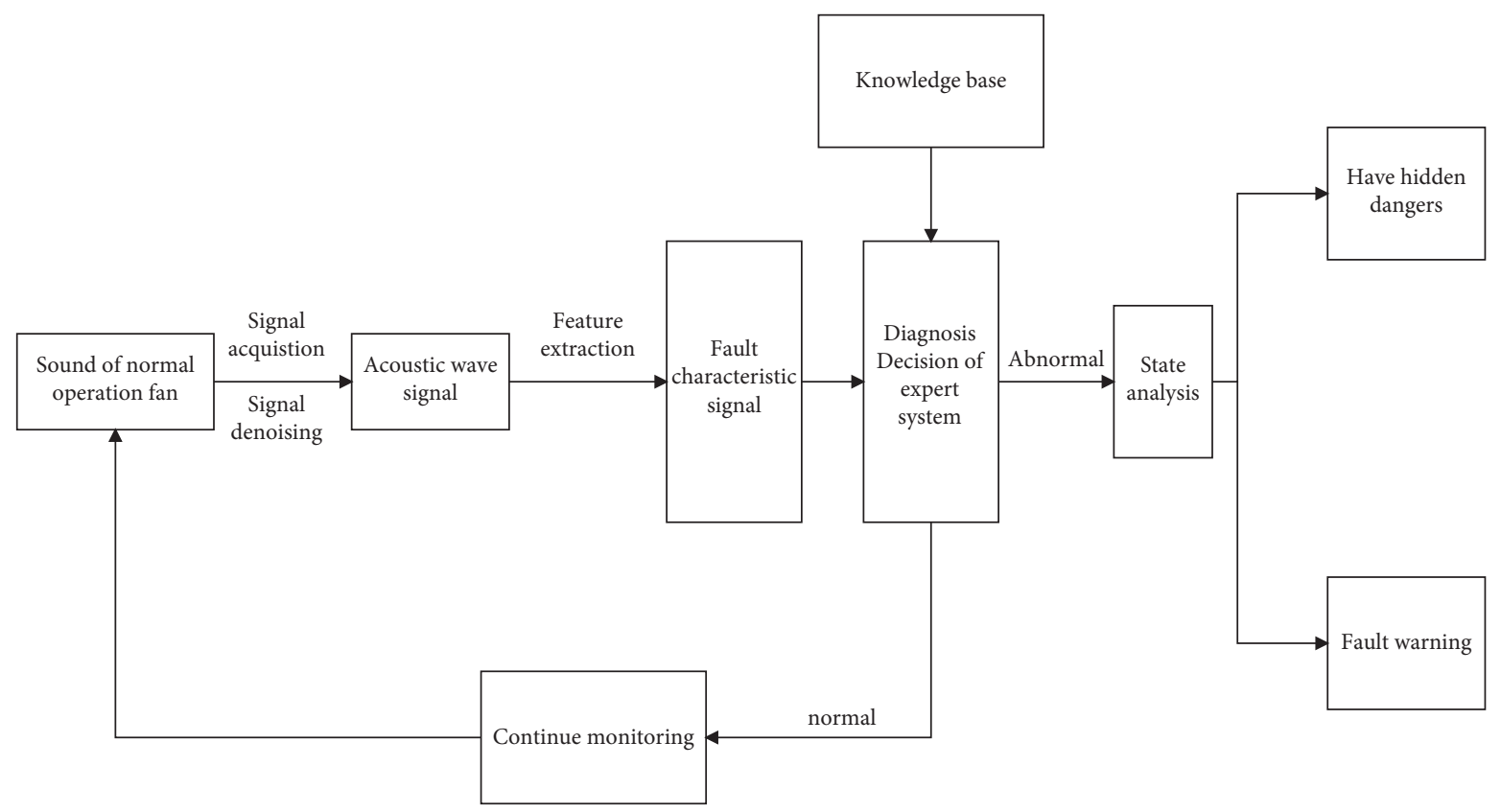

FIGURE 1: Fault diagnosis system diagram of mine fan.

This phenomenon is more obvious when the width or thickness of the blade increases. The frequency of noise is composed of multiple frequencies, which are related to the speed of the fan.

3.1.2. Noise of Blade Vortex. During the operation of the fan, the vortex will be generated on the back of the moving wing, which will not only reduce the efficiency of the fan, but also produce noise. During the operation of the fan, the vortex will be generated on the back of the moving wing, which will not only reduce the efficiency of the fan, but also produce noise.

3.1.3. Noise of Turbulence. When the air is flowing, if it meets sharp obstacles, it is easy to cause turbulence. Although the turbulence is different from the vortex, it will also produce noise or high-frequency whistling, which will also cause efficiency loss to the fan. Although the turbulence is different from the vortex, it will also produce noise or highfrequency whistling, which will also cause efficiency loss to the fan.

3.1.4. Noise of the Air Duct Housing Resonates. The joint between the air duct and the inner surface of the fan shell shall be smooth to avoid roughness and unevenness, which may cause tearing sound. Moreover, because the connected pipeline will produce resonance, the subtle sound will become larger, resulting in greater noise. In the design, sometimes the air duct can be covered with antisound material to reduce noise.

3.1.5. Noise Outside the Fan. In addition to the fixed noise of the fan itself, there are many noise sources, such as the bearing due to insufficient precision, improper assembly, or poor maintenance, which will cause abnormal noise. The motor also produces noise, some of which is caused by poor design or poor manufacturing quality control, but sometimes by the cooling fans inside and outside the motor. Gears and belts also produce noise due to friction. The noise produced by the resonance of other structures cannot be ignored, which is sometimes caused by the imbalance of the body.

3.2. Signal Acquisition and Analysis. Sound signal carries all kinds of information. The purpose of sound signal processing is to extract these kinds of information. The processing methods can be divided into time-domain analysis and frequency domain analysis. The time-domain analysis is relatively intuitive and simple, while the frequency domain analysis changes the sound signal from the time domain to the frequency domain composed of sine function or cosine function to extract the characteristic parameters.

In this paper, the sound equipment with sampling frequency of $44100 \mathrm{~Hz}$ is used to sample the local fan in the mine. The time-domain waveform and frequency spectrum of the fan in normal operation are obtained by processing the audio signal collected from the field. The sampling point is $4.4 * 104$, and the time domain waveform is shown in Figures 2(a) and 2(b), which reflects the image of the sound signal in the time domain, and the relationship between the change of the sound signal with time and the amplitude of the sound signal. The time-domain signal is changed to the frequency domain through Fourier change, and the spectrum components and the intensity of each component of the signal are analyzed. It reflects the relationship between the sound signal frequency $(\mathrm{Hz})$ and the amplitude of the sound signal at this frequency. From the analysis results, it can be seen that the fan audio signal is composed of multiple frequencies, the main frequency components are within $8000 \mathrm{~Hz}$, and the 


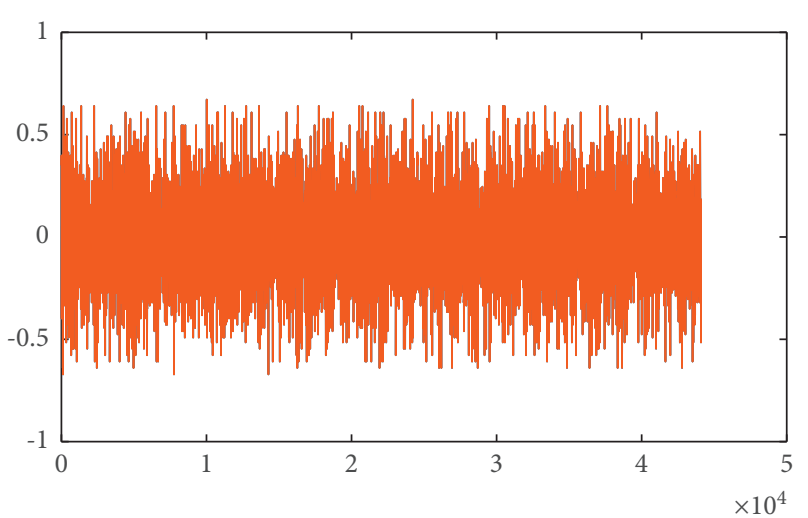

(a)

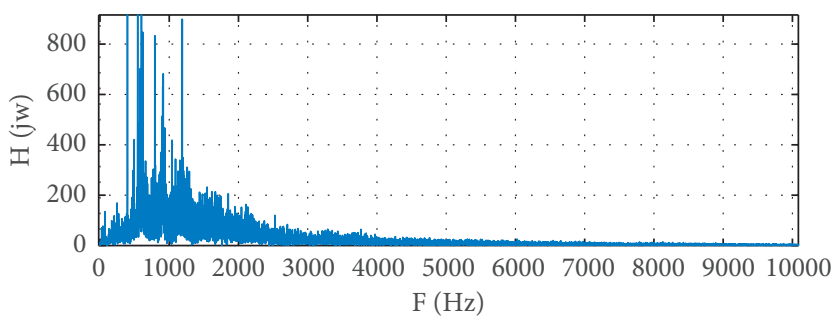

(b)

Figure 2: (a) Time-domain waveform of signal. (b) Spectrum of signal.

$200 \mathrm{~Hz}, 300 \mathrm{~Hz}$, and $600 \mathrm{~Hz}$ frequency points in the power spectrum show obvious current spectrum.

3.3. Wavelet Denoising. Because the fault signal is a transient signal, the traditional Fourier transform analysis method cannot be distinguished. This is because the use of Fourier transform analysis will be able to reflect the fault characteristics of the local signal in the entire frequency domain smoothed out, thus losing the useful information used to analyze the fault, resulting in large errors. The wavelet analysis has good time-frequency localization, using different scales, in different widths of the time window for analysis, especially suitable for the processing of odd signals $[10,11]$. The principle of wavelet threshold denoising is to generate a threshold value by estimating the noise intensity and use the threshold function to process the wavelet coefficients to remove part of the noise-related components, so as to increase the proportion of useful signals. There are three main steps: firstly, the noisy signal is decomposed into several layers of wavelet coefficients by wavelet variation, then the noise-related components are removed by processing the wavelet coefficients in each layer using the threshold function, and finally the signal is reconstructed by wavelet inverse transform [12].

(1) Choose a wavelet, determine the number of layers $M$ of wavelet decomposition, find suitable wavelet basis, and then use discrete wavelets to perform $M$-layer wavelet decomposition on noise-bearing signals [13]. The Harr wavelet function is defined as follows:

$$
\psi(x)=\left(\begin{array}{ll}
1, & 0 \leq x<\frac{1}{2}, \\
-1, & \frac{1}{2} \leq x<1, \\
0, & \text { others. }
\end{array}\right.
$$

(2) Threshold quantization is performed on the highfrequency coefficients of each layer from the first layer to $M$. Soft and hard thresholding methods are commonly used [14]. The soft thresholding method sets the wavelet coefficients smaller than the threshold to zero and makes the wavelet coefficients larger than the threshold shrink toward zero, with the following expressions:

$$
s=\left(\begin{array}{ll}
x, & |x|>T, \\
0, & |x| \leq T .
\end{array}\right.
$$

The two methods differ from each other, with the former having continuity and being mathematically easy to handle, and the latter being closer to the implementation case.

The key to threshold processing is the selection of the threshold value. If the threshold value is too small, noise will remain after noise cancellation; if the threshold value is too large, important signals will be filtered out and cause bias.

(3) Wavelet reconstruction of the signal based on the low-frequency coefficients of the $M$ th layer of the wavelet decomposition and the high-frequency coefficients of the first to $M$ th layers after quantization is performed with the following expression [15]:

$$
c_{j-1}, n=\sum_{n} c_{j}, n h_{k}-2_{n}+\sum_{n} d_{j}, n g_{k}-2_{n} .
$$

$c_{j, n}$ is the scale factor; $d_{j}$ is the wavelet coefficients; $h$, $g$ are a pair of orthogonal mirror filter sets; $j$ is the number of decomposition layers; $n$ is discrete sampling points.

Wavelet transform has good time-frequency characteristics. Compared with traditional linear filtering and nonlinear filtering, wavelet denoising has the characteristics of low entropy, multiresolution, decorrelation, and diversity of wavelet base selection. Reduce the entropy of the signal after transformation, and better characterize the nonstationary characteristics of the signal, such as edge, peak, and breakpoint. The noise tends to whiten after transformation. At the same time, wavelet transform can 
flexibly select different wavelet bases according to the characteristics of the signal.

3.4. Elimination of Measured Signal Noise. MATLAB software is used to denoise the measured sound signal of $5.5 \mathrm{~kW}$ mine press in axial flow local fan. Through the denoising function in the wavelet toolbox, the measured audio signal is decomposed into five layers by using the Harr wavelet in the wavelet toolbox to obtain Figure 3(a), in which $s$ is the original signal, $A 5$ is the fifth-order approximation signal, $d 1-d 5$ is the detail signal after wavelet decomposition, and the frequency components are arranged from low to high [16].

Denoise the signal and get the image (the red part is the original signal and the purple part is the denoised signal). It can be seen that the burr in the signal can be eliminated by denoising, and the rich high-frequency information can still be retained, which has obvious denoising effect. The characteristics of wavelet transform multiresolution analysis make it have a strong advantage in noise reduction rate [17].

\section{Wavelet Packet-Based Feature Extraction}

Feature extraction refers to the identification and separation of faults in the process of extracting the characteristic information related to wind turbine faults from the state signal. The general feature quantity is selected in order to transform the information from the measurement space to the feature space that is built for a substantial reduction in the number of dimensions, thus highlighting the fault characteristics and improving the accuracy for subsequent fault diagnosis. There are usually 3 steps to extract feature quantities $[18,19]$.

(1) The signal is decomposed by 3 layers of wavelet packets, and the signal features of 8 frequency components from low to high frequencies are extracted in layer 3, respectively. These 8 wavelet packet coefficients summarize the features of the wind turbine audio signal in different frequency bands. Its wavelet packet decomposition tree is shown in Figure 4. The wavelet packet coefficients of each node are obtained as $\left[S_{3,0}, S_{3,1}, S_{3,2}, S_{3,3}, S_{3,4}\right.$, $\left.S_{3,5}, S_{3,6}, S_{3,7}\right]$.

(2) Find the total energy of the signal in each frequency band. Let the energy corresponding to $S_{3 j b e} E_{3 j}, j=0$, $1, \ldots, 7$; then we have

$$
E_{3 j}=\left|\int S_{3 j}(t)\right|^{2} \mathrm{~d} t=\sum_{k=1}^{n}\left|x_{j k}^{2}\right|,
$$

where $x_{j k}(j=0,1, \ldots, 7, k=1,2, \ldots, n)$ denotes the amplitude of the discrete points of the reconstructed signal $S_{3 j}$.

(3) Construct the eigenvectors. Construct the eigenvector $T$ with energy as an element: $T=[E 30, E 31$, E32, E33, E34, E35, E36, E37,].
In practical engineering, when the energy is relatively large, it can cause inconvenience during data analysis. Therefore, the energy value of the signal is usually normalized, and the ratio of the energy of each node to the total energy is taken as a feature to form the corresponding feature vector [20]. The corresponding equation is

$$
T(j, i)=\frac{E(j, i)}{\sum_{k \in(j, i)} E(j, i)} .
$$

$T(j, i)$ is the percentage of energy of the corresponding node; $E(j, i)$ is the energy value of the corresponding node. The above calculation results in a new feature vector $T^{\prime}=$ $[T 0, T 1, T 2, T 3, T 4, T 5, T 6, T 7$, ] for the wind turbine audio.

Collect the audio signals of five groups of fans under normal operation, which are $G 1, G 2, G 3$, G4, and G5, respectively. The duration of the audio signal is $1 \mathrm{~s}$. Each group of audio signals is divided into eight energy nodes ( $T 0, T 1$, $T 2, T 3, T 4, T 5, T 6, T 7)$. Use the wavelet packet of $\mathrm{db} 3$ of MATLAB wavelet packet tool for three-layer decomposition to obtain the ratio of the energy of each node to the total energy, as shown in Figure 5. After obtaining the total energy of the signal, the energy of each node is obtained $\left(E_{30}, E_{31}\right.$, $\left.E_{32}, E_{33}, E_{34}, E_{35}, E_{36}, E_{37}\right)$, as shown in Table 1. From the energy proportion in Figure 5 , it can be seen that the proportion of each node in the total energy has no obvious change. From the output energy of the signal in Table 1, it can be seen that the output energy of each node has a small change. From the two aspects, it can be seen that the test data has good repeatability, so the requirement for the number of tests $n$ is not too much. Finally, according to the feature vector of the signal, through the fault diagnosis expert system for decision-making classification, we can judge whether the mine fan is faulty or not.

By collecting the sound signal of the mine fan, wavelet noise eliminated. Extract the feature vector of the signal to get several groups of data that can characterize the signal information by using MATLAB. This is used as the judgment basis of inference machine [21, 22].

\section{Fault Signal Simulation}

Due to the low failure rate of mine fan, it is easy to collect the audio signal in the normal operation state, but it is very difficult to study the audio signal in the specific fault type. Therefore, in order to study the fan audio signal under some specific fault conditions, the audio processing software is used for simulation. In this paper, three kinds of fan fault audio signals are simulated. When the fan is running under load, the fan bearing overheats, and the fault sound is "buzzing." When the bearing is abnormal, the fault phenomena are various abnormal sounds, such as sharp sound, whistling sound, or impact sound. When the operation of the belt is abnormal, the fault phenomenon is that the belt jumps out during the operation and makes a "pop" sound, and the speed of the belt pulley decreases. Based on the above audio signal denoising and feature extraction algorithm, five groups of data of three kinds of fault audio signals are analyzed, and their corresponding energy is calculated as shown in Figure 6. 


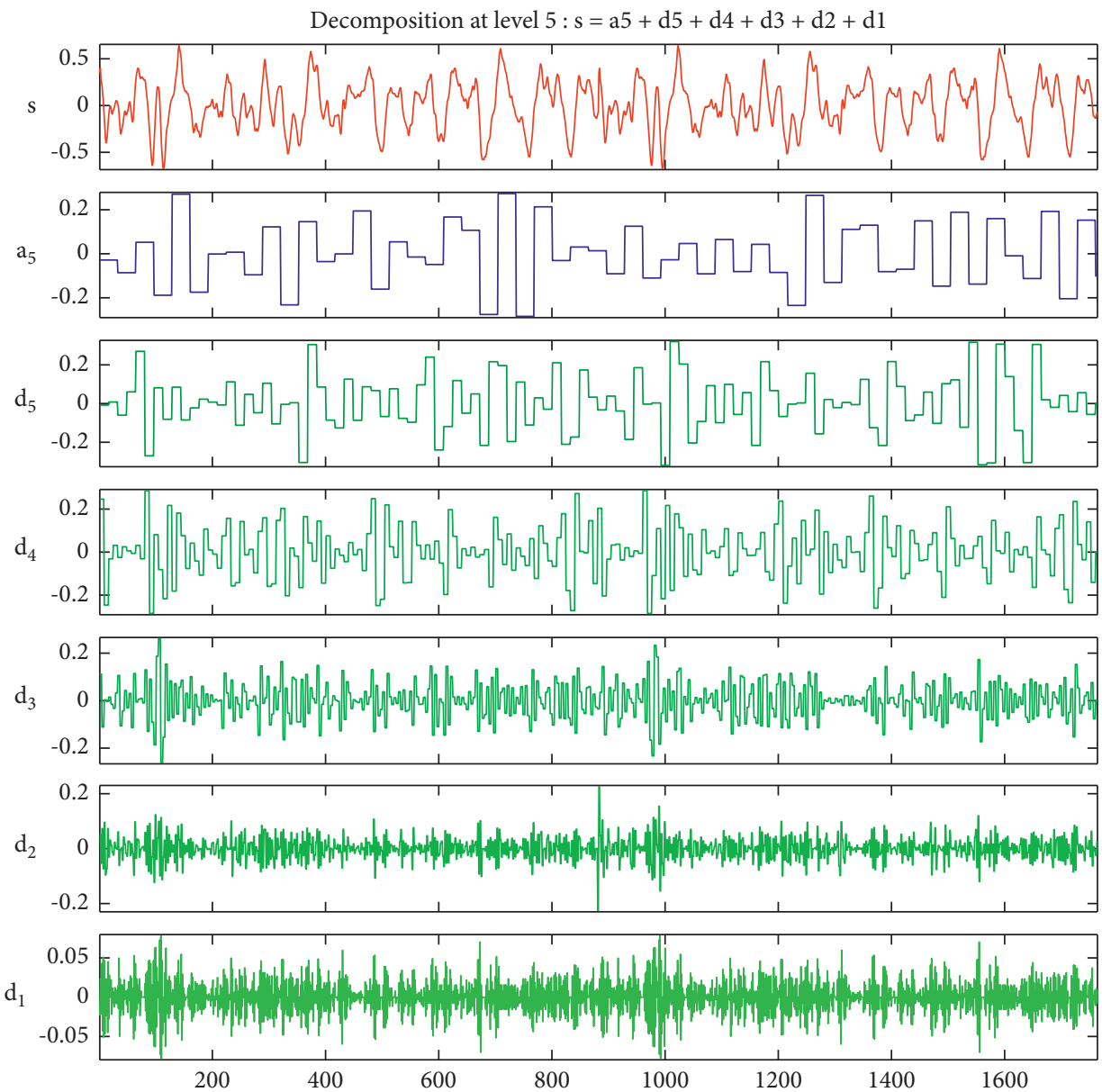

(a)

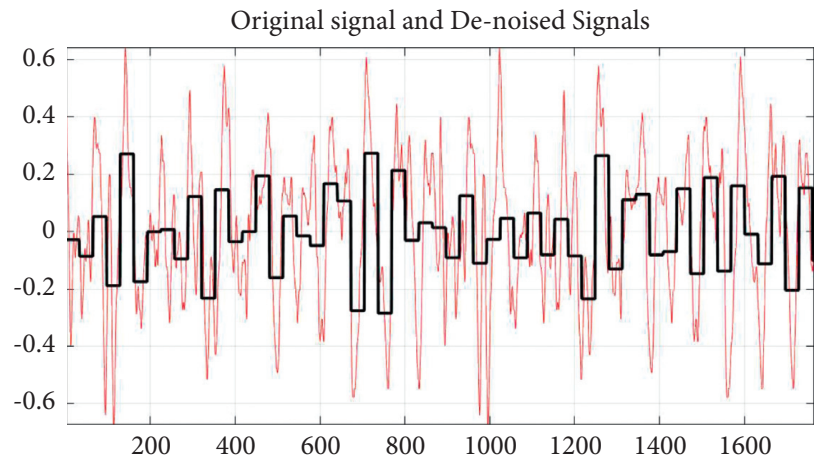

(b)

Figure 3: (a) Original signal decomposition diagram. (b) Comparison of original signal and denoised signal.

Figure 6(a) simulates the sound of the fan under load. Simulate five groups of audio signals in this state, namely, $G 1, G 2, G 3, G 4$, and $G 5$, and calculate the proportion of each node energy to the total energy. Similarly, Figures 6(b) and 6(c) are audio signals under the fault state of fan bearing and the abnormal state of fan belt. Different types of fault audio signals simulate 5 groups of signals and extract characteristic information. It is not difficult to find that the fan has corresponding frequency characteristics under different fault operating states, and the characteristic frequencies of audio signals with the same fault have high similarity. The characteristic frequencies of different fault types change greatly, and these fault characteristic frequencies are also quite different from the frequency of normal operation state, which can be used as the basis of fault reasoning. Once the test audio signal has different characteristic frequencies, the fault can be judged, and the reliability of sound monitoring is verified. However, due to the limited research data, the threshold between different states cannot be accurately determined, which needs further research. 


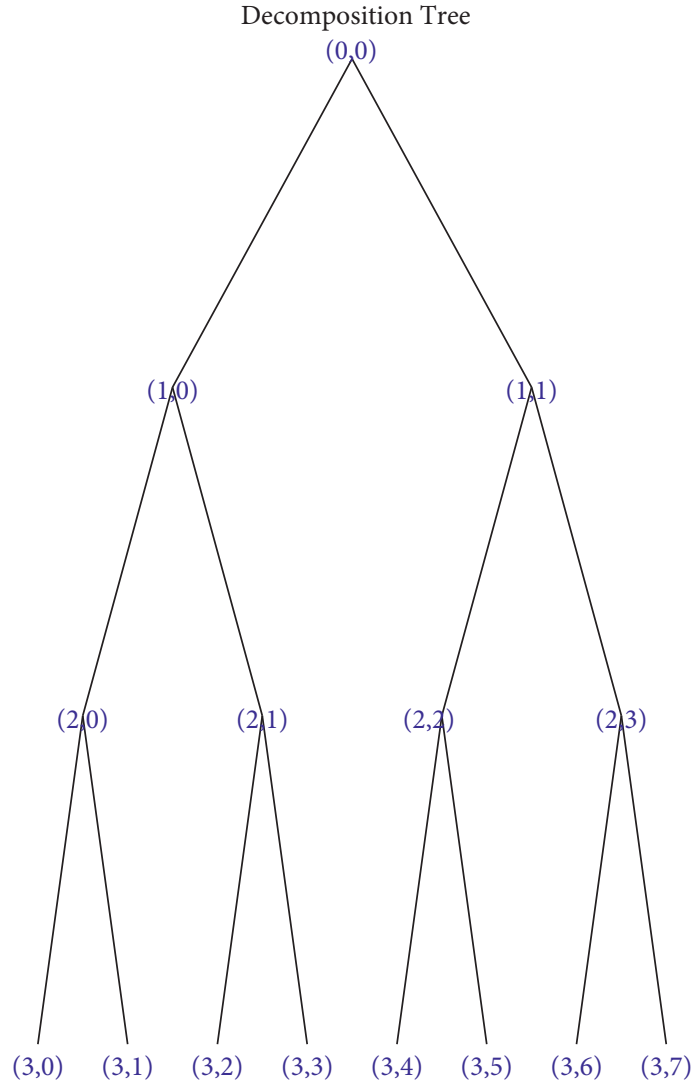

Figure 4: Wavelet decomposition tree.

PROPORTION OF ENERGY

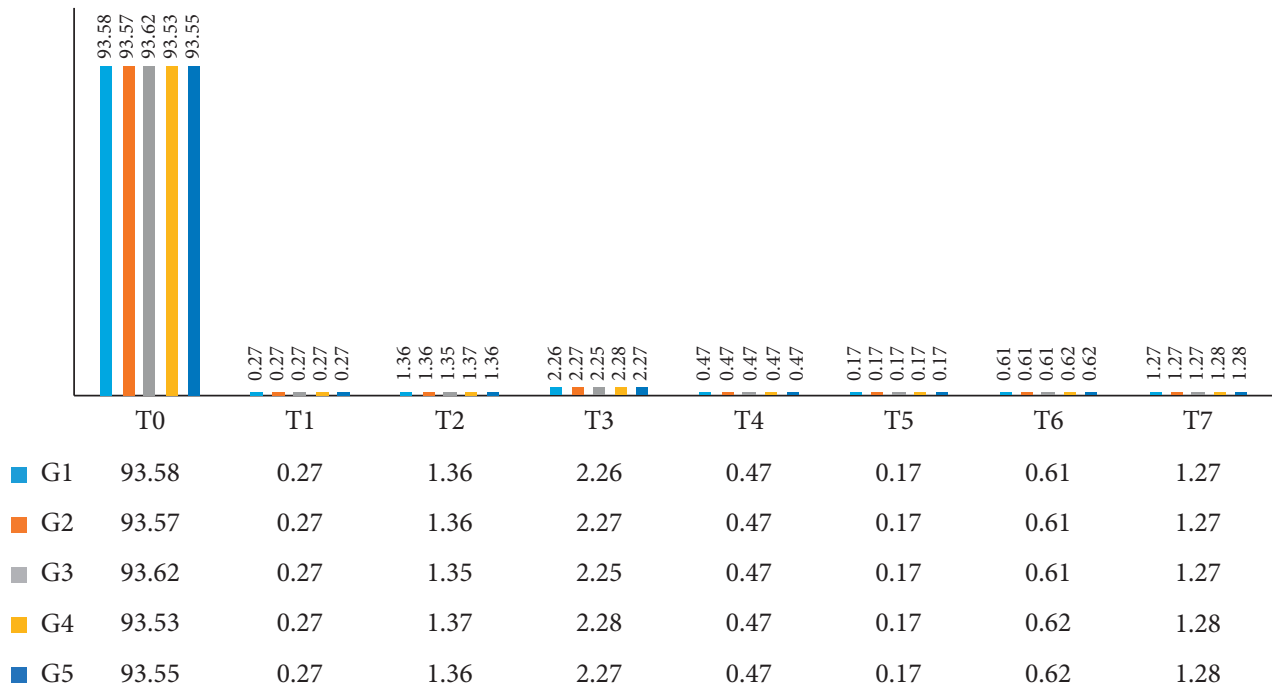

FIgURE 5: Energy proportion. 
TABLE 1: The output energy of the signal table.

\begin{tabular}{lcccccccc}
\hline Group & $E_{30}$ & $E_{31}$ & $E_{32}$ & $E_{33}$ & $E_{34}$ & $E_{35}$ & $E_{36}$ & $E_{37}$ \\
\hline 1 & 12590.25 & 36.32 & 182.97 & 304.06 & 63.23 & 22.87 & 82.06 & 170.86 \\
2 & 12268.89 & 35.4 & 178.32 & 297.64 & 61.62 & 22.29 & 79.98 & 166.52 \\
3 & 12462.69 & 35.94 & 179.71 & 299.52 & 62.56 & 22.63 & 81.2 \\
4 & 12864.11 & 37.13 & 188.42 & 313.59 & 64.64 & 23.38 & 85.27 & 169.06 \\
5 & 12794.83 & 36.92 & 186 & 310.46 & 64.28 & 23.25 & 84.79 & 176.05 \\
\hline
\end{tabular}

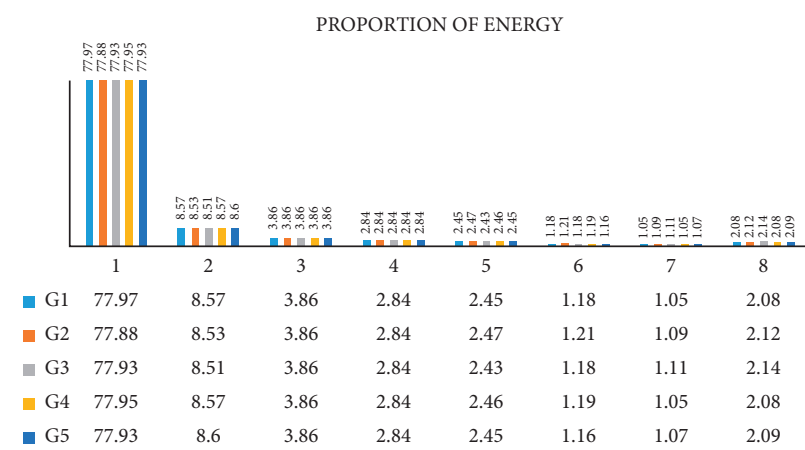

(a)

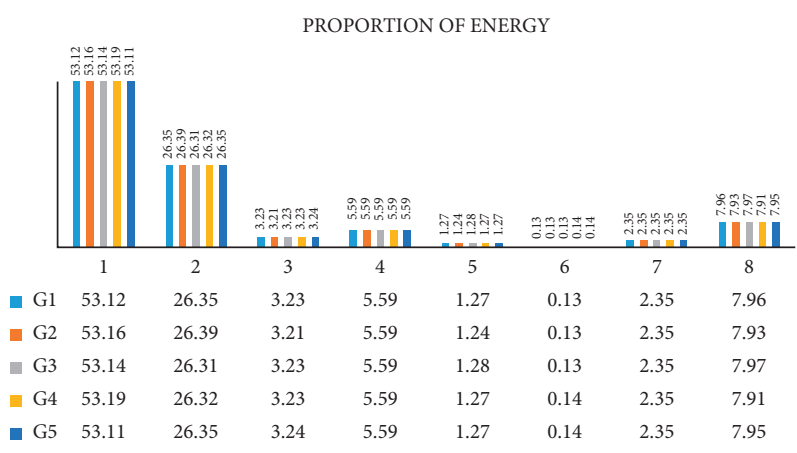

(b)

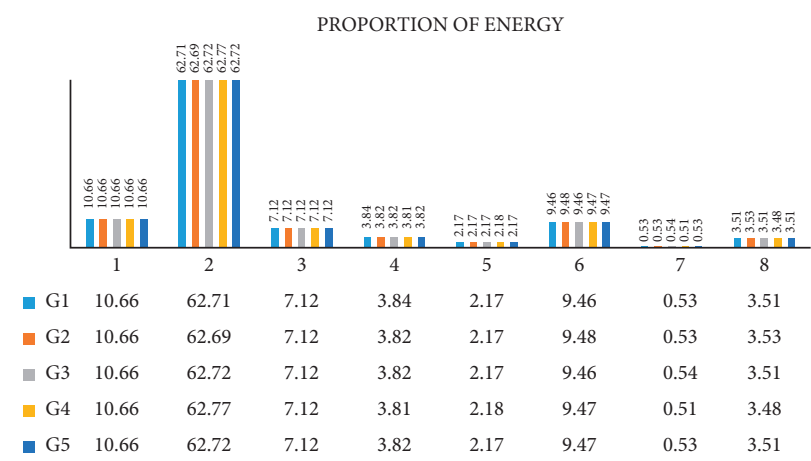

(c)

Figure 6: (a) Under fan load condition. (b) Fan bearing fault. (c) Under abnormal condition of fan belt.

\section{Conclusion}

This paper puts forward a fault diagnosis scheme based on the sound signal of mine fan and analyzes the noise source of mine fan, including the noise generated by blade rotation, the noise generated by blade eddy current, the noise generated by turbulent flow, the noise generated by resonance with the air duct shell, and the noise caused outside the fan. Through the audio data collected by the audio device, the wavelet denoising is used to filter the signal layer by layer, and the noise in the signal is reconstructed to retain its mutation characteristics. The wavelet packet algorithm is used to quantify the time-domain information. According to the output energy and energy proportion of each node, the feature vector is used for the diagnosis and decision-making of reasoning machine. The results show that the method highlights the fault characteristics and can judge whether there are hidden faults in the mine fan. Through the audio processing software to simulate the sound signal of the fan in three fault states, using the above data analysis, the feature vector of the fault state signal is extracted. It is found that different fault states have their corresponding feature frequencies, which are obviously different from the feature vector of the fan in normal operation, which can be used as the basis for the fault signal identification of the expert system.

The fault diagnosis based on sound analysis is a new noncontact online monitoring method, which can accurately reflect the operation status of electrical equipment without affecting the normal operation of electrical equipment. It is more effective for online monitoring and fault diagnosis of mine fan. This method can ensure the normal operation of the mine fan, find the hidden danger of the fan in time, and ensure the life safety of the underground workers, which is of great significance to promote the digital mine.

\section{Data Availability}

The sound signal data used to support the findings of this study are included within the article. 


\section{Conflicts of Interest}

The authors declare that they have no conflicts of interest.

\section{Acknowledgments}

The authors acknowledge the support from the Science Research Foundation of Wuhan Institute of Technology (K201854).

\section{References}

[1] L. Yao and L. Pan, "The application of nonlinear modeling in the fault diagnosis of fan," Advanced Materials Research, vol. 926-930, p. 3181, 2014.

[2] W. P. Hong, M. J. Liao, Application of fruit fly optimization algorithm-least square support vector machine in fault diagnosis of fans," Advanced Materials Research, vol. 860-863, p. 2912, 2014.

[3] F. Shu, "Application of improved support vector machine in fault diagnosis of mine fan," Mining machinery, vol. 34, no. 3, pp. 39-41, 2006.

[4] Z. Xuan and X. Li, "HHT extraction of vibration signal characteristic components of mine fan," Journal of Hefei Union University, vol. 38, no. 1, pp. 34-38, 2016.

[5] R. Zhai ., "Research on fault diagnosis and maintenance technology of coal mine electromechanical equipment," China Petroleum and chemical industry standard and quality, vol. 40, no. 16, pp. 21-22, 2020.

[6] H. Zhao, "Fault prediction of key components of wind turbine based on BP neural network," Power station system engineering, vol. 37, no. 2, pp. 21-22, 2021.

[7] W. Chang, Xi Peng, Q. Luo, B. Zeng, X. Jin, and L. Hu, "Fault analysis of catalytic main fan based on condition monitoring and fault diagnosis technology," China Equipment Engineering, no. 5, pp. 152-154, 2021.

[8] L. Pan, S. Zhao, and B. Li, "A new method for fault diagnosis of electrical equipment based on acoustic signal analysis," Electric power automation equipment, vol. 29, no. 8, pp. 87-90, 2009.

[9] J. Chen, Research on Abrasive belt Wear Condition Monitoring Method Based on Grinding Sound Signal Characteristics, Shanghai Jiaotong University, Shanghai, China, 2018.

[10] Y. Liu and G. Zhao, "Wavelet fault detection method based on filter preprocessing," Power automation equipment, no. 2, pp. 51-55, 2007.

[11] H. Zhao, Da Zhang, J. Yang, fancui Meng, and M. Zhang, "Application of wavelet stratification in laser Doppler velocimetry," Laser Technology, vol. 43, no. 1, pp. 103-108, 2019.

[12] J. Zhang, Q. Zhong, and Y. Dai, "Determination of decomposition level and threshold in application of wavelet threshold denoising method," Chinese Journal of electrical engineering, vol. 24, no. 2, pp. 119-123, 2004.

[13] X. Wang, J. Lian, S. Fei, and Z. Zhang, "Chaotic multiple regression daily runoff prediction model based on wavelet denoising," Journal of System Simulation, no. 15, pp. 36053608, 2007.

[14] Y. Qin, S. Huang, and Q. Zhao, "Chaotic time series prediction model based on wavelet denoising and LS-SVM and its application," Geodesy and geodynamics, vol. 28, no. 6, pp. 96-100, 2008.
[15] Li Wen, Z. Liu, and Y. Ge, "Several methods of wavelet denoising," Journal of Hefei University of Technology, no. 2, pp. 167-172, 2002.

[16] J. Ren, "Research on data analysis method based on wavelet transform," Science and technology innovation, no. 16, pp. 39-40, 2019.

[17] yunyun Zheng, S. Chen, and Y. Hu, "Application of wavelet transform and BP neural network model in settlement deformation monitoring," Surveying and mapping and spatial geographic information, vol. 42, no. 2, pp. 101-103 + 107, 2019.

[18] D. Xu, "AC sampling signal denoising technology based on wavelet transform," Electric power automation equipment, no. 10, pp. 15-17, 2001.

[19] X. Liu, H. Liu, Y. Chen, and dingfang Ke, "Classification method of transient power quality based on optimal wavelet packet basis decomposition," Power automation equipment, no. 10, pp. 36-39, 2005.

[20] J. Chen, "Research on fault diagnosis method of traction transformer based on sound signal analysis," Electrical application, vol. 39, no. 2, pp. 25-29, 2020.

[21] G.-l. Feng, B.-r. Chen, Q. Jiang, Y.-x. Xiao, W.-j. Niu, and P.-x. Li, "Excavation-induced microseismicity and rockburst occurrence: s," vol. 28, no. 2, , pp. 582-594, Journal of Central South University, 2021.

[22] G.-L. Feng, X.-T. Feng, B.-r. Chen, Y.-X. Xiao, and Y. Yu, "A microseismic method for dynamic warning of rockburst development processes in tunnels," Rock Mechanics and Rock Engineering, vol. 48, no. 5, pp. 2061-2076, 2015. 